\title{
A Compact and Repetitively Triggered, Field- distortion Low-jitter Spark-gap Switch
}

\author{
Falun Song, Fei Li, Beizhen Zhang, Mingdong Zhu, Chunxia Li, Ganping Wang, Haitao \\ Gong, Yanqing Gan, Xiao Jin, B. M. Novac, I. R. Smith
}

\begin{abstract}
This paper describes the development of a small field-distortion spark gap switch. Comprehensive experimental studies made with the switch revealed its self-breakdown characteristics, voltage operating range, time delay and jitter, repetition frequency and finally the electrode erosion processes and duration of its service life time. A series of design improvements were required to be implemented in the switch design. For example, to reduce the structural inductance of the switch and to improve its working characteristics, the main electrode was designed as a circular track structure. Similarly, to increase the operation stability and reduce the self-breakdown probability, the trigger electrode was designed as a disk-like structure. Moreover, to balance the gas pressure in the discharge region and increase the stability during closure, a circular hole was added at the center of the trigger electrode. The compact switch has a small size of only $150 \mathrm{~mm} \times 42$ $\mathrm{mm}$, a weight of $1.5 \mathrm{~kg}$ and can successfully be operated at a voltage over $110 \mathrm{kV}$, at a repetition rate between $1 \mathrm{~Hz}$ to $50 \mathrm{~Hz}$ and having a jitter of less than 4 ns. It was experimentally demonstrated that during normal operation conditions, the switch life time exceeds 100,000 shots.
\end{abstract}

Index Terms - field distortion, spark gap switch, repetition frequency, low jitter, electrode erosion, lifetime

\section{INTRODUCTION}

$I^{1}$ $\mathrm{N}$ recent years, important civilian and military applications were all based on pulsed power sources similar to the one described in the paper [1]-[3]. The closing switch represents a key component of such units and its performance directly affects the overall technical specification of the power source [4]. Although in recent years semiconductor switches made impressive advances, gas spark gap switches are still the best choice to meet the needs of high-voltage and high-current required in a compact high-power pulsed generator. Along the years extensive research have been conducted on spark gap switches for

Falun Song, Fei Li, Beizhen Zhang, Chunxia Li, Ganping Wang, Haitao Gong, Yanqing Gan and Xiao Jin, are with Science and Technology on High Power Microwave Laboratory, Institute of Applied Electronics, China Academy of Engineering Physics, Mianyang 621900, China (email: songfalun@caep.cn; shanxilifei@163.com; 99477228@qq.com; lichunxia@tsinghua.org.cn; wanggpcaep@163.com; 2712019073@qq.com; emplasma@tom.com; Xiao_jin@hotmail.com). (Corresponding author: Falun Song). enabling their application in various domains [5]-[13]. For a single-shot or low repetition-rate operation two, three, or multielectrode products with good performance at high peak current (1-500 kA) and high peak voltage (2.5-100 kV) are all commercially available, such as the T-Series spark gap switches produced by L3 company [14] and the SG-Series spark gap switches produced by R. E. Beverly III \& Associates [15]. Related to Linear Transformer Drivers (LTDs) a series of low inductance, low jitter, and high current gas switches have also been extensively studied [16]-[20]. However, with the need to develop compact, mobile and high repetition rate pulsed power sources, the technical requirements for such switches are becoming more and more demanding [21]. As an example, a typical Marx generator for high-power microwave applications requires a small volume design, operation with a low jitter at both high peak voltage $(\sim 100 \mathrm{kV})$ and high peak current $(\sim 10 \mathrm{kA})$ at a repetition rate of up to $100 \mathrm{~Hz}$, while at the same time having a long service-life [22]-[24]. For such type of applications, to the authors' best knowledge, the corresponding switches have not been presented in the open literature.

A small field distortion spark gap switch was developed in an attempt to obtain optimum operation parameters for applications requiring very compact, repetitive and low jitter design. Although the breakdown mechanism of the field distortion spark gap switch has been extensively studied, the breakdown mechanism under high repetition-rate operation has many complex differences when compared with single-shot operation and therefore represents a serious theoretical challenge [25-28]. This paper attempts to experimentally provide all the working characteristics of a small field distortion switch, such as selfbreakdown voltage, jitter, repetition frequency, and lifetime.

It is well-known that the electrode erosion of the switch is a key factor influencing its operating characteristics, stability and service life [29]-[36]. Therefore, the erosion characteristic of

B. M. Novac and I. R. Smith are with Wolfson School of Mechanical, Electrical and Manufacturing Engineering, Loughborough University, Loughborough, Leicestershire LE11 3TU, United Kingdom (email: B.M.Novac@lboro.ac.uk; i.r.smith@lboro.ac.uk).

Mingdong Zhu is with China State Key Laboratory of Advanced Welding and Joining, Harbin Institute of Technology, Harbin 150001, China (email: zhumingdong2008@163.com) 
the switch is also experimentally studied in this paper, providing a basis for improving the performance of the switch.

\section{DESIGN OF A SMALL FIELD DISTORTION SPARK GAP SWITCH}

\section{A. Structural design}

A small field distortion spark gap switch was designed as presented in Fig. 1. The main components include two main electrodes, two plate insulators of Polymethyl methacrylate (PMMA), a disk-like trigger electrode, two pairs of O-ring seals and sixteen nylon screws for fastening. The main electrodes are made of $316 \mathrm{~L}$ stainless steel and both have a circular track structure which helps reducing the switch self-inductance, thus improving its $\mathrm{di} / \mathrm{dt}$ characteristics. At the same time, under repetitive operation, the structure helps in dispersing the breakdown to different regions, which is beneficial in improving the service-life. The inner side of plate insulator has annular grooves to increase the path of the electric surface breakdown, thereby increasing the operational voltage. Due to the strong electric field operation, in the triple-point regions of the switch cavity the angle $\theta$ between the main electrode and the plate insulator varies (Fig. 1), depending on the electric field strength, thereby minimizing the influence of the corona effects on the insulator. In order to further increase the working stability and reduce the self-breakdown probability, the trigger electrode is designed as a disk-like structure with a circular hole provided at the center of the trigger disk to ensure that a uniform gas pressure exists on both sides of the trigger electrode during the discharge process, thereby improving the switch reliability.

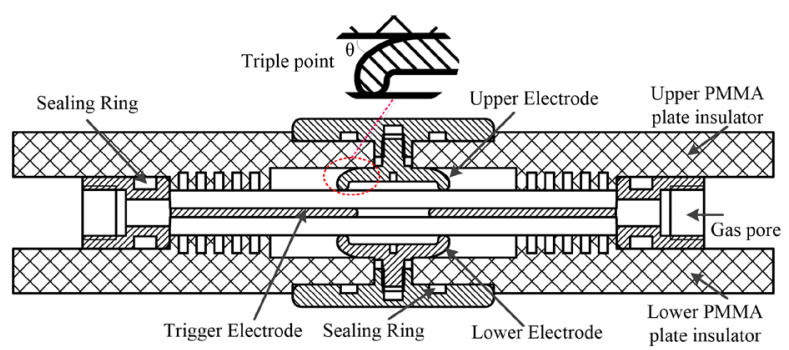

Fig. 1. Schematic of the compact three-electrode spark gap switch. Details of the triple-point region are also shown.

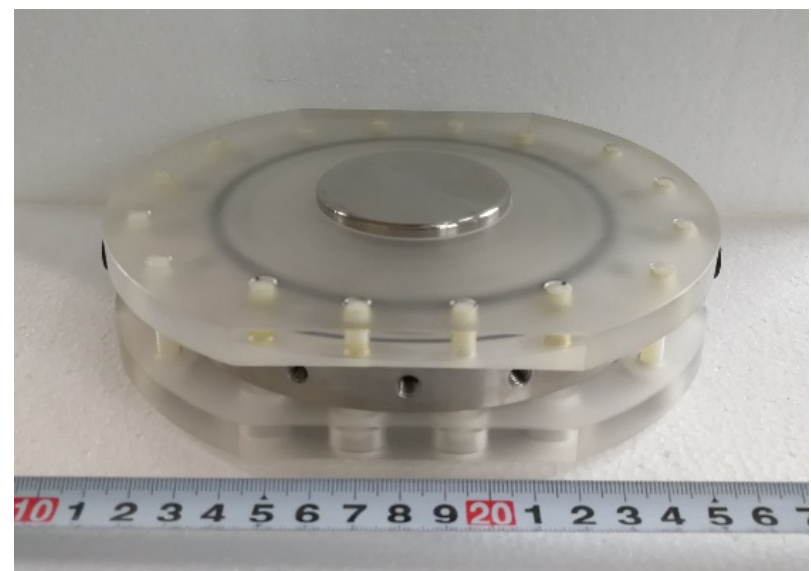

Fig. 2. Photo of the compact field distortion switch assembly.
The main electrodes and the plate insulators are sealed using a pair of O-rings. The upper and lower plate insulators and the trigger electrode are also sealed by a pair of O-rings and fastened by 16 nylon screws. The distance between the main electrode and the trigger electrode is $4 \mathrm{~mm}$. Fig. 2 shows a photo of the field distortion switch with the outer diameter, height and weight being 150 $\mathrm{mm}, 42 \mathrm{~mm}$ and $1.5 \mathrm{~kg}$, respectively.

\section{B. Electric field analysis}

The distribution of the electric field inside the switch is determined by the geometry of the main electrodes and does not change as a function of the applied voltage. The calculated electric field distribution is presented in Fig. 3 for $+50 \mathrm{kV}$ applied on the upper and $-50 \mathrm{kV}$ applied on the lower electrode. Fig. 3(a) demonstrates that the electric field is mainly distributed in the region between the upper and lower electrodes, with a peak field of $21.8 \mathrm{kV} / \mathrm{mm}$ generated on the surface of the ring electrode in a circular region having a diameter of about $1 \mathrm{~mm}$. The electric field enhancement factor is calculated as 1.74 .
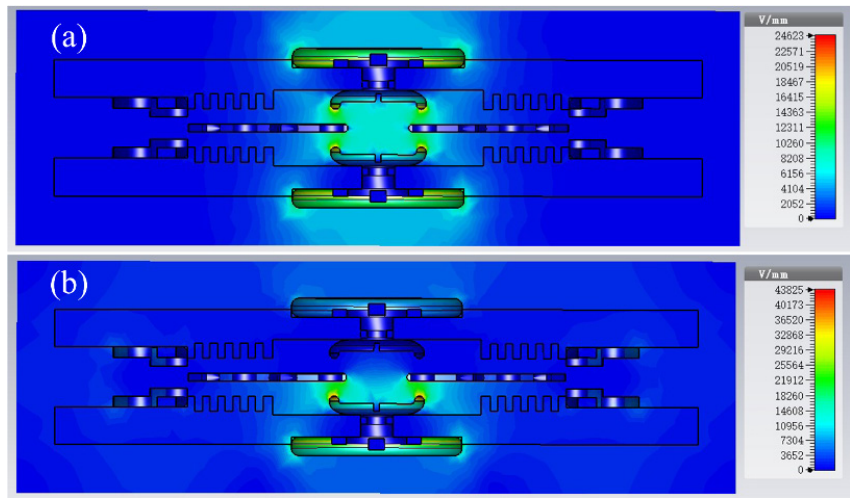

Fig. 3. Electric field distribution inside and around the switch for: a) no trigger voltage applied and $b$ ) when a voltage is applied on the trigger electrode

Fig. 3(b) shows that when a voltage of $+50 \mathrm{kV}$ is applied to the trigger electrode, the electric field distribution is changing. A peak electric field of $43.6 \mathrm{kV} / \mathrm{mm}$ is now located in the region between the negative electrode and the trigger electrode, corresponding to an increased field enhancement factor of 3.48. Under these conditions an electric breakdown across the gap is easily achieved, which is beneficial to the operating characteristics of the switch.

As Fig. 3 also shows, the electric field strength outside the switch is relatively high around the electrodes. Therefore, to avoid corona effects and unwanted external discharges, the switch must be operated under transformer oil.

\section{EXPERIMENTAL SETUP}

The schematic of the experimental arrangement used in the present studies is shown in Fig.4 and includes three main parts: a power supply, the main discharge circuit containing the switch and a measurement system.

For repetitive experiments, the HV charger is based on a series resonant constant current power supply, generating a maximum output voltage of $\pm 60 \mathrm{kV}$ at a peak repetition frequency of $200 \mathrm{~Hz}$. For static voltage breakdown tests, a high voltage DC power supply with a maximum output voltage of $\pm 100 \mathrm{kV}$ was used. The time rate-of-change of the charging voltage was $17 \mathrm{kV} / \mathrm{ms}$ for the constant current power supply and $50 \mathrm{kV} / \mathrm{s}$ for the DC power supply. 
The pulse trigger source is a high-voltage pulse generator based on a hydrogen thyristor with an output voltage of $50 \mathrm{kV}$, having a time rate-of-change of $0.25 \mathrm{kV} / \mathrm{ns}$ at a peak repetition rate of 200 $\mathrm{Hz}$.

The main discharge loop circuit consists of two, series-connected, $100 \mathrm{kV} 35 \mathrm{nF}$ pulse capacitors, with the field-distortion spark gap switch placed between the two capacitors (Fig. 4) and a $5 \Omega$ high-power ceramic resistor used as a resistive load. In order to obtain a high peak current, the components in the main discharge circuit are closely connected, with the total self-inductance of the circuit being only $220 \mathrm{nH}$. The trigger source and the power supply are software controlled using a PC.

The measurement system consists of:

- $\quad$ two high-voltage probes (EP-150k, NEP), measuring respectively the output voltage of the trigger source and the voltage across the resistive load, connected to a four-channel digital storage oscilloscope (Lecroy HDO 6054) by 30-meter-long coaxial cables

- a high definition electron microscope (VHX-5000, Keyence) for studying the microstructure of the switch electrode and insulator surfaces

- an X-ray photoelectron spectroscope (ThermoFischer, ESCALAB 250Xi) for performing X-ray photoelectron spectroscopy (XPS) for analyzing the chemical composition of the switch insulator surfaces before and after the tests.

As mentioned in [37], a $\mathrm{SF}_{6}-\mathrm{N}_{2}$ gas mixture has more efficient stabilization characteristics than pure $\mathrm{SF}_{6}$ and can be triggered more easily over a wider pressure range than either pure $\mathrm{SF}_{6}$ or $\mathrm{N}_{2}$. Therefore, although for static voltage breakdown tests $\mathrm{SF}_{6}, \mathrm{~N}_{2}$ and a $\mathrm{SF}_{6}-\mathrm{N}_{2}$ gas mixture were all used, only $\mathrm{SF}_{6}-$ $\mathrm{N}_{2}$ gas mixture was used during the experimental studies for obtaining the main switch characteristics.

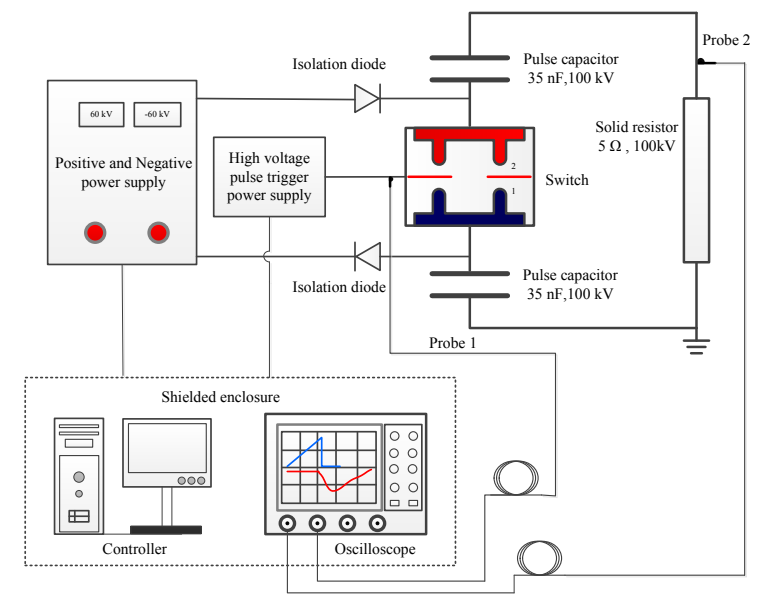

Fig. 4. Schematic of the experimental arrangement used in the studies.

\section{EXPERIMENTAL STUDIES}

\section{A. Switch forming and self-breakdown tests}

After the experimental assembly was completed and the experimental testing just begun, it was evident that the stability of the switch was not sufficiently good. During the first few hundred shots, a period of switch 'forming', it was observed that the breakdown voltage greatly varied and the time delay had a too large jitter. The reason for these unwanted phenomena was found to be residual burrs or/and incompletely cleaned stains remaining on the surface of the electrodes after they were manufactured. Due to erosion however, after the switch has been operated hundreds of times, the surface of the electrode changes: the burrs are greatly reduced and the stains are vaporized under high temperature or carried away by the molten electrode material. Even better, the microstructure of the electrodes also changes in time and the surfaces become uneven, resulting in many field enhancement points. These in turn can increase the efficiency of initial electrons generation during the conduction phase, reduce the statistical delay and improve the overall stability of the switch.

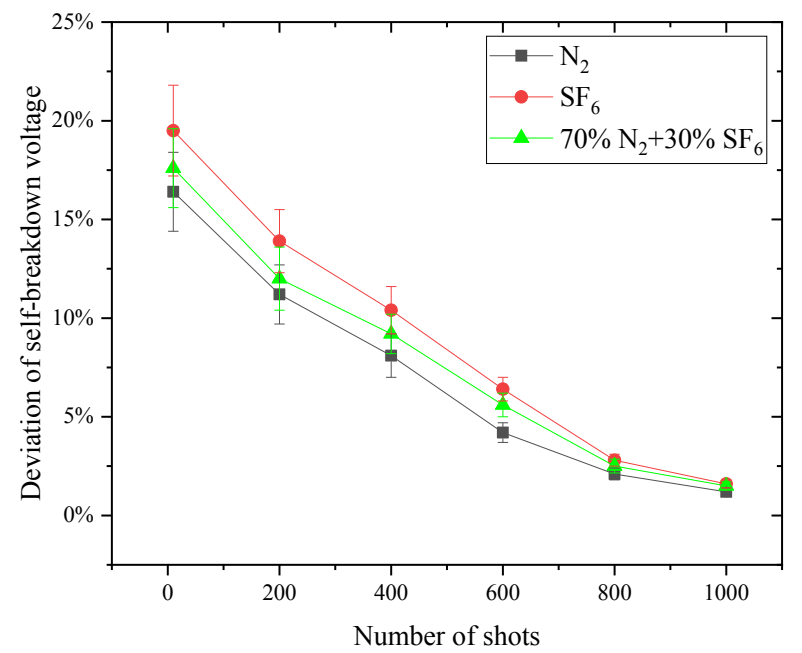

Fig. 5. The deviation of static self-breakdown voltage varies with the number of shots during the switch 'forming'.

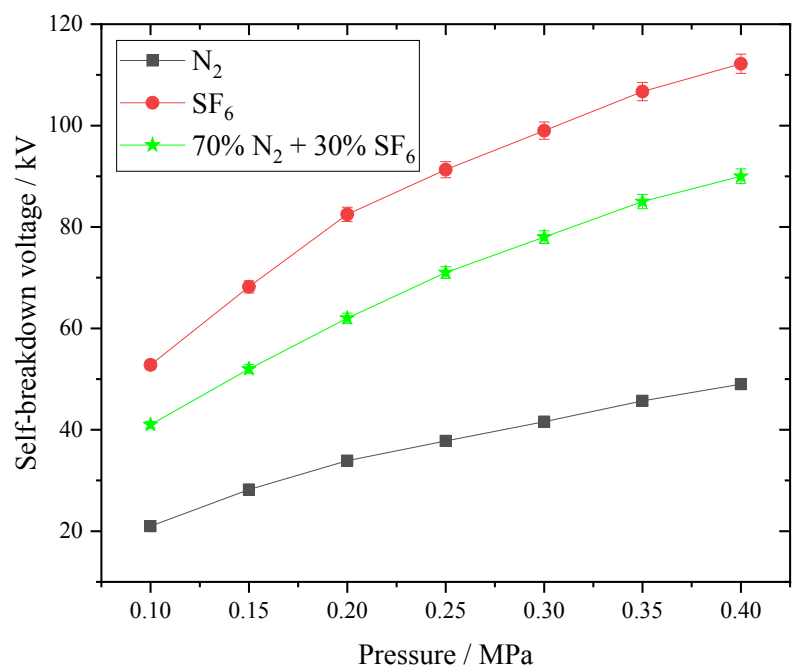

Fig. 6. Self-breakdown voltage after switch 'forming' versus gas pressure for various gases.

Fig. 5 shows the variation of the static self-breakdown voltage during the switch 'forming' process, tests made at an absolute pressure of $0.1 \mathrm{MPa}$. As can be noticed, during the first ten self-breakdown shots the breakdown voltage varied dramatically. The standard deviations of the self-breakdown voltage of $\mathrm{SF}_{6}, \mathrm{~N}_{2}$ and the mixture for the first ten shots are $19.5 \%, 16.4 \%$, and $17.6 \%$, respectively. After 800 self-breakdown shots however, the changes 
in the self-breakdown voltage became stable and their percentage changes in amplitude are all less than $3 \%$.

After 1,000 shots, the switch 'forming' is completed and the corresponding relationship between the self-breakdown voltage and the gas pressure is presented in Fig. 6. For the same gas pressure of $0.4 \mathrm{MPa}$, the switch has the highest withstand voltage $(112 \mathrm{kV})$ when filled with $\mathrm{SF}_{6}$, the value being 2.4 times higher than when filled with $\mathrm{N}_{2}$ and about 1.28 times higher than when a gas mixture of $70 \% \mathrm{~N}_{2}$ and $30 \% \mathrm{SF}_{6}$ is used.

\section{B. Voltage operating range}

The voltage operating range of the switch under triggered conditions is an important characteristic. In order to study the voltage operating range of the switch, a series resonant high-voltage constant current power supply was used to achieve millisecond fast charging. When the switch is filled with the $70 \% \mathrm{~N}_{2}$ and $30 \% \mathrm{SF}_{6}$ gas mixture, the relationship between the operating voltage and the gas pressure is as presented in Fig. 7. The minimum controllable voltage is the minimum voltage applied on the high-voltage electrode for which the trigger pulse can still trigger the switch while the maximum controllable voltage is the maximum voltage on the high-voltage electrode for which the switch can be stably triggered without any chance of a self-breakdown. The data in Fig. 7 clearly demonstrates the switch has a wide voltage operating range. When the gas pressure in the switch cavity is $0.1 \mathrm{MPa}$, the voltage operating range of the switch is between $31 \%$ and $90 \%$ of the pulsed self-breakdown voltage. When the gas pressure is raised to 0.4 $\mathrm{MPa}$, the voltage operating range is however only between $42 \%$ and $85 \%$ i.e., by increasing in the gas pressure the operating range decreases. The explanation is that a lower pressure corresponds to lower insulation strength and in such conditions it is easier to trigger the switch, a phenomenon also observed in [8].

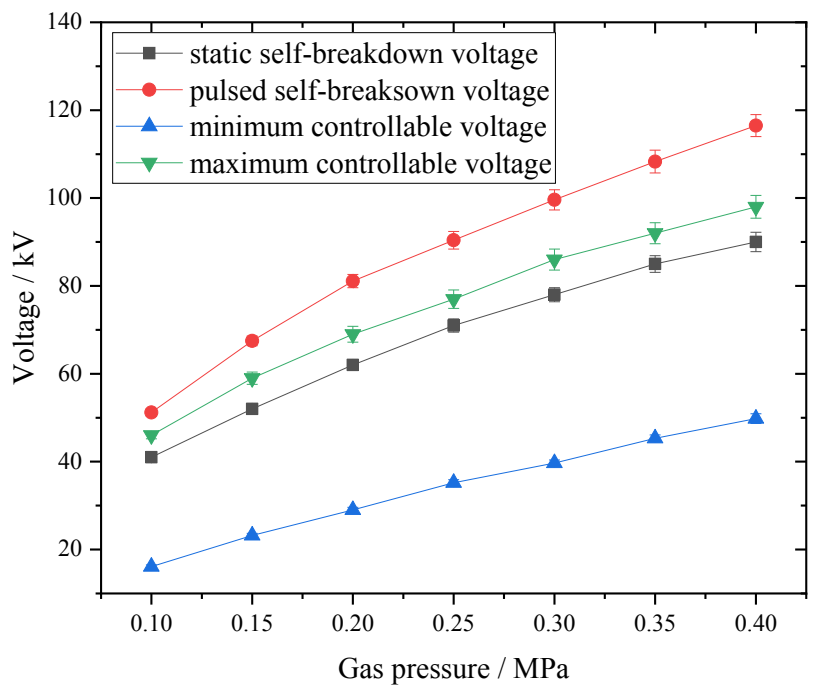

Fig. 7. Operating voltage range of the switch versus gas pressure when the switch is filled with $70 \% \mathrm{~N}_{2}$ and $30 \% \mathrm{SF}_{6}$ gas mixture.

\section{Time delay and jitter}

The time delay and jitter characteristics are closely related to the electrode structure, the filling gas, the operating voltage and the trigger pulse. Fig. 8 shows the variation of the time delay with the ratio of the operating voltage to self-breakdown voltage at different gas pressures, using a trigger pulse with a voltage time rate-of-change of $0.25 \mathrm{kV} / \mathrm{ns}$ and with the switch filled with $\mathrm{SF}_{6}-\mathrm{N}_{2}$ gas mixture. As Fig. 8 demonstrates, the higher the gas pressure in the switch cavity, the longer is the breakdown time delay. This is because as the gas pressure increases, the self-breakdown voltage also increases, resulting in an increase in the required trigger pulse voltage. For the same trigger pulse the breakdown time delay will also increase.

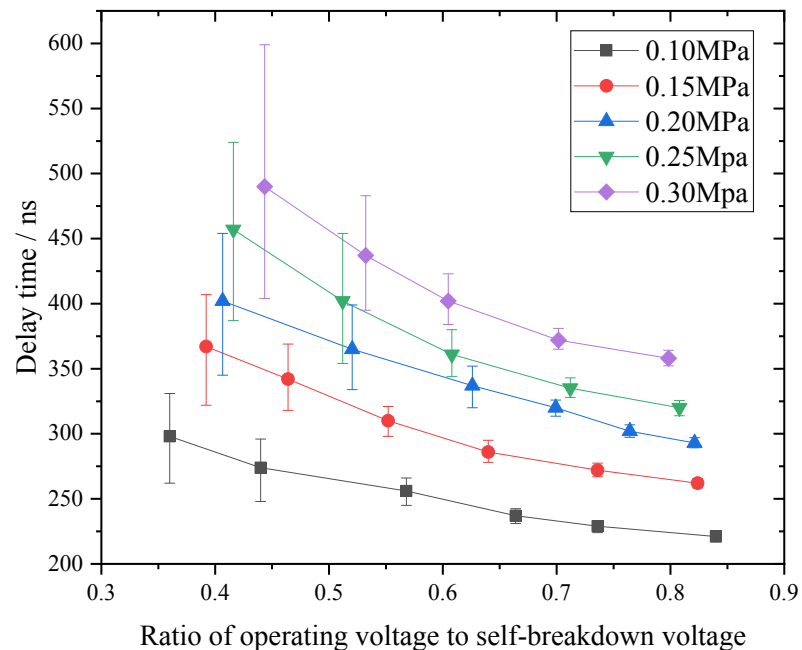

Fig. 8. Delay time varies with the ratio of operating voltage to self-breakdown voltage at different gas pressures, the tests used a trigger pulse with a voltage time rate-of-change of $0.25 \mathrm{kV} / \mathrm{ns}$.

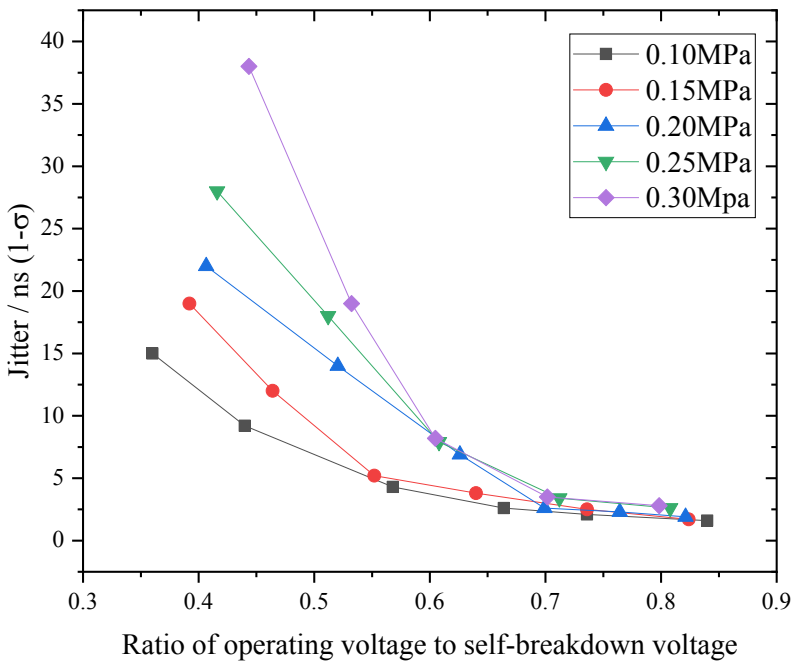

Fig. 9. The time jitter varies with the ratio of operating voltage to self-breakdown voltage at different gas pressures. $\sigma$ represents the standard deviation.

When the filling gas pressure is maintained constant, the breakdown time delay decreases with an increase in the working voltage ratio. This is because as the electric field inside the switch increases, more initial electrons are generated, which decreases the statistical time delay of the switch. Under the action of the strong electric field the velocity of the current streamer between the switch electrodes is also increased, which correspondingly reduces the time delay of the discharge formation.

Fig. 9 demonstrates the time jitter decreases with an increase of the ratio of operating voltage to self-breakdown voltage at different gas pressures. When the operating voltage ratio is less than 0.6 , the time jitter is greater than $5 \mathrm{~ns}$, regardless of the gas pressure. However, when the operating voltage ratio is greater than 0.7 , the time jitter of the switch becomes less than 3 ns and converges to a minimum value. 
Fig. 10 presents the variation of the time-delay with the number of shots at a repetition frequency rate of $30 \mathrm{~Hz}$ for $10 \mathrm{sec}-$ onds. It can be seen that the pressure in the switch cavity increased from the initial $0.25 \mathrm{MPa}$ to $0.32 \mathrm{MPa}$, which undergoes a process from rapid increase to gradual stabilization. However, the time-delay and the jitter did not increase with the number of shots, but show a slight decrease. These phenomena can be explained as following.

Due to the Joule heating generated by the discharge current, the temperature $T$ in the switch cavity increases. According to the equation of state for an ideal gas, $\mathrm{PV}=\mathrm{N} k_{b} T$ (where $\mathrm{k}_{b}$ is the Boltzmann constant) and assuming the volume of the switch cavity $\mathrm{V}$ and the number of gas molecules $\mathrm{N}$ remain both constant, the gas pressure $\mathrm{P}$ must increase. As the gas density $\rho=\mathrm{NM} / \mathrm{V}$ (where $\mathrm{M}$ is the molar mass) remains constant, the breakdown voltage remains the same (i.e., the insulation strength of a gas depends basically on the gas density). However, as the temperature of the gas rises, the corresponding increase in the kinetic energy of the gas molecules increases the number of ionized molecules, so that the time-delay and the jitter both decrease slightly.

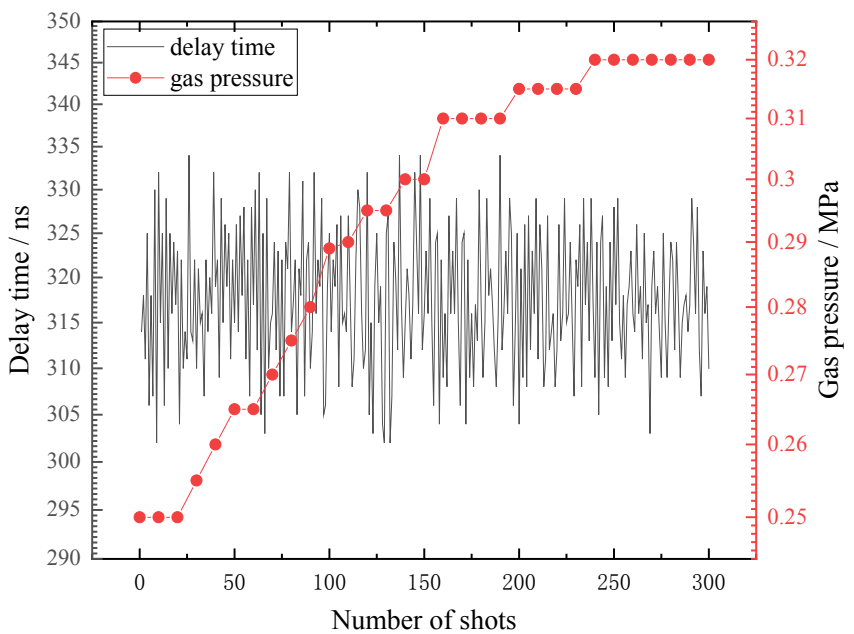

Fig. 10. Variation of the delay time and gas pressure with the number of shots

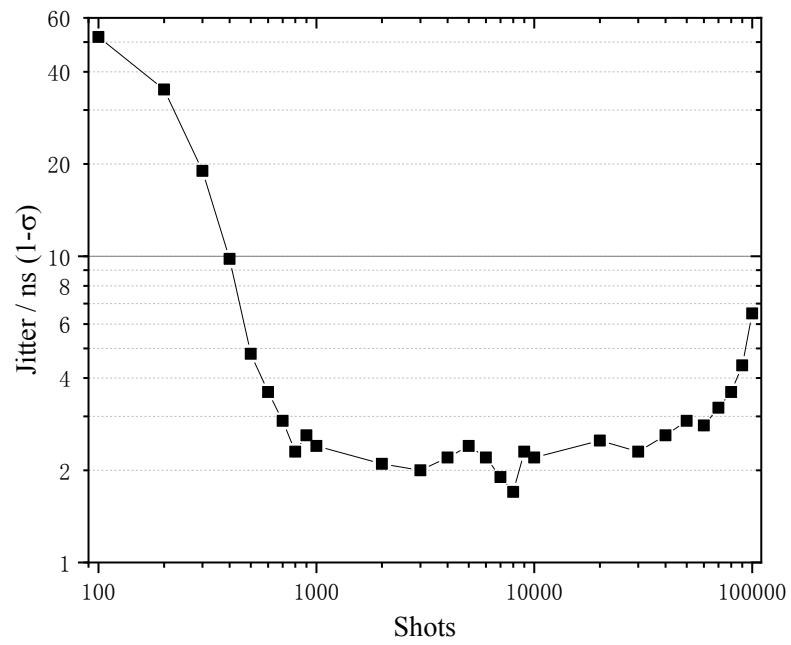

Fig. 11. Relationship between the time jitter and the number of shots.

Fig. 11 shows the relationship between the time jitter and the number of shots, when the switch is filled with the $\mathrm{SF}_{6}-\mathrm{N}_{2}$ gas mixture at a pressure of $0.3 \mathrm{MPa}$, the applied voltage is $\pm 35 \mathrm{kV}$ and the current is $8.5 \mathrm{kA}$. During testing of the switch lifetime, the discharge sequence was paused many times. Each pausing time was sufficiently long to allow the temperature inside the switch cavity to drop down to the initial (ambient) temperature. This procedure actually mimics the conditions the switch is operated during practical repetitive pulsed power source applications where a continuous burst is allowed for only 5 minutes for which, at a repetition rate of $30 \mathrm{~Hz}$, the total number of discharges is limited to 9,000 before a pause is taken.

The tests results show the time jitter is rapidly reduced within the first 700 shots. For the next 60,000 shots, the time jitter is practically stable between $1.7 \mathrm{~ns}$ and $3.0 \mathrm{~ns}$. However, from about 70,000 shots to 100,000 shots the electrode erosion starts to play a role and the time jitter increases from $3.2 \mathrm{~ns}$ to $6.5 \mathrm{~ns}$. The reasons for this phenomenon are detailed below.

\section{Switch operation at a high repetition rate frequency}

Fig. 12 presents the variation of the time jitter with the repetition rate frequency, for experiments performed with the switch filled with a $\mathrm{SF}_{6}-\mathrm{N}_{2}$ gas mixture at a pressure of $0.3 \mathrm{MPa}$ and with the ratio of the operating voltage to the self-breakdown voltage being 0.8 . It can be seen that when the switch operates at a repetition rate of no more than $50 \mathrm{~Hz}$, the time jitter is very stable and varies only between $2.0 \mathrm{~ns}$ and $4.0 \mathrm{~ns}$. However, when the repetition frequency is higher than $100 \mathrm{~Hz}$, the time jitter increases rapidly and the switch operation becomes unstable. The explanation for this phenomenon is that at a too high repetition rate frequency produces an increased Joule heating effect and under such conditions the dynamics of the gas components in the switch cavity does not allow recovering its normal initial state required for a stable operation. Basically, the tests proved that it takes more than $10 \mathrm{~ms}$ for the gas to recover its stable initial conditions.

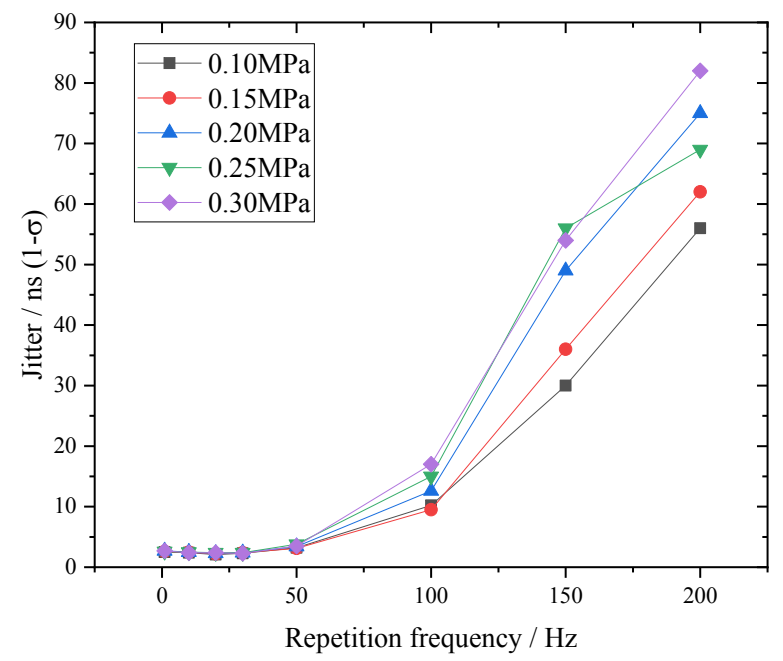

Fig. 12. Variations of the time jitter with the repetition rate frequency for various filling pressures.

\section{E. Electrode erosion complex phenomena and their influence on the switch service-time}

Fig. 13 presents photographs of the main switch components: trigger electrode, plate insulator and main electrode, after 100,000 shots. It can be seen that an annular erosion region is formed on the trigger electrode, with a diameter similar to that 
of the main electrode. This annular region is evenly eroded, which is an important indication suggesting a stable operation.

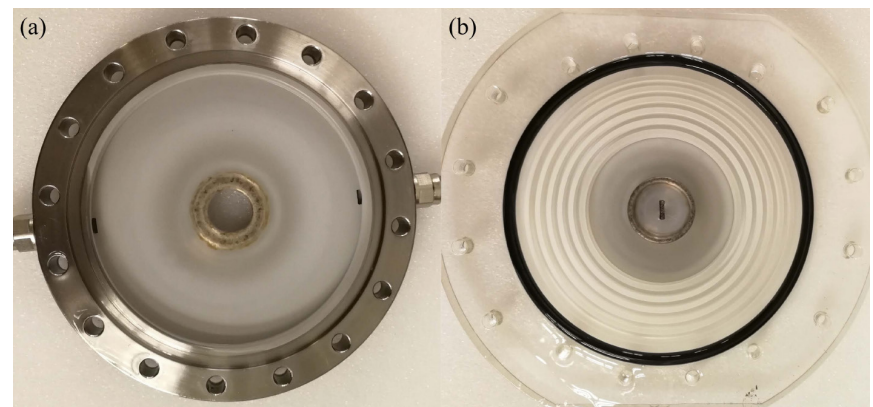

Fig. 13. Photographs of some switch components after 100,000 shots. (a) trigger electrode and (b) plate insulator and the main electrode.

However, there are many tiny cracks, pits and bumps on both the main electrode and the trigger electrode surface and also a layer of white powder attached to the surface of both the trigger electrode and the plate insulator. The appearance of all these unwanted phenomena may provide the explanation of why the time jitter gradually increases after 70,000 shots. In order to fully understand the nature of these phenomena, the microstructure of the main electrode, the trigger electrode and the plate insulator surfaces were all analyzed, with the main results presented in Fig. 14.

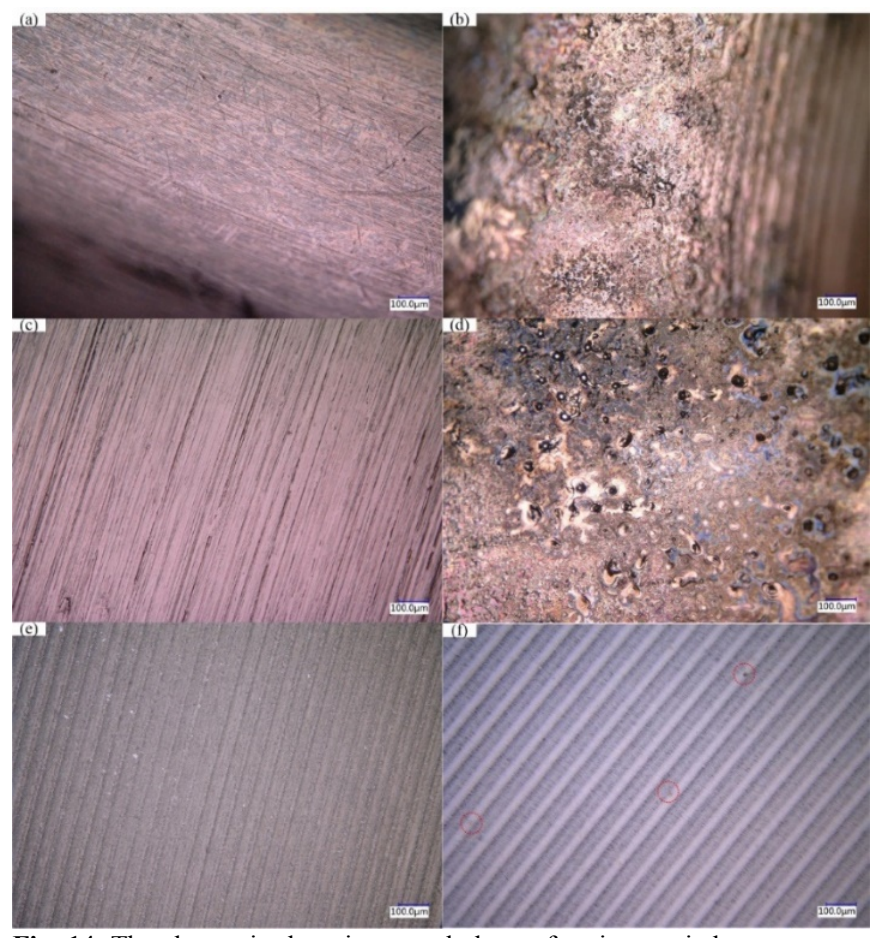

Fig. 14. The change in the micromorphology of various switch components from 0 shots to 100,000 shots. (a) initial state of the main electrode surface. (b) main electrode surface after 100,000 shots. (c) initial state of the main discharge area of the trigger electrode. (d) main discharge area of the trigger electrode after 100,000 shots. (e) initial state of the first annular surface on the insulating plate. (e) the first annular surface on the insulating plate after 100,000 shots; the position of a few metallic micro particles adhered to the insulator surface is highlighted using circles.

On the metallic electrode surface, corresponding to the discharge regions, a large number of protrusions and micro pores are noticeable, with a great number of crater-like holes of 20 $50 \mu \mathrm{m}$ diameter generated on the flat surface of the trigger electrode, all these defects are presented in both Fig. 14 (b) and (d).
On the surface of the plate insulator there are now some 10 $\mu \mathrm{m}$-sized sputtered metal spots, as shown in Fig. 14(f), which proves that melted electrode material near the electric arc spot was ejected during high frequency operation. These metallic micro particles adhered to the surface of the plate insulator, obviously decreasing its insulation properties and leading to an overall reduction of the service-life of the switch.
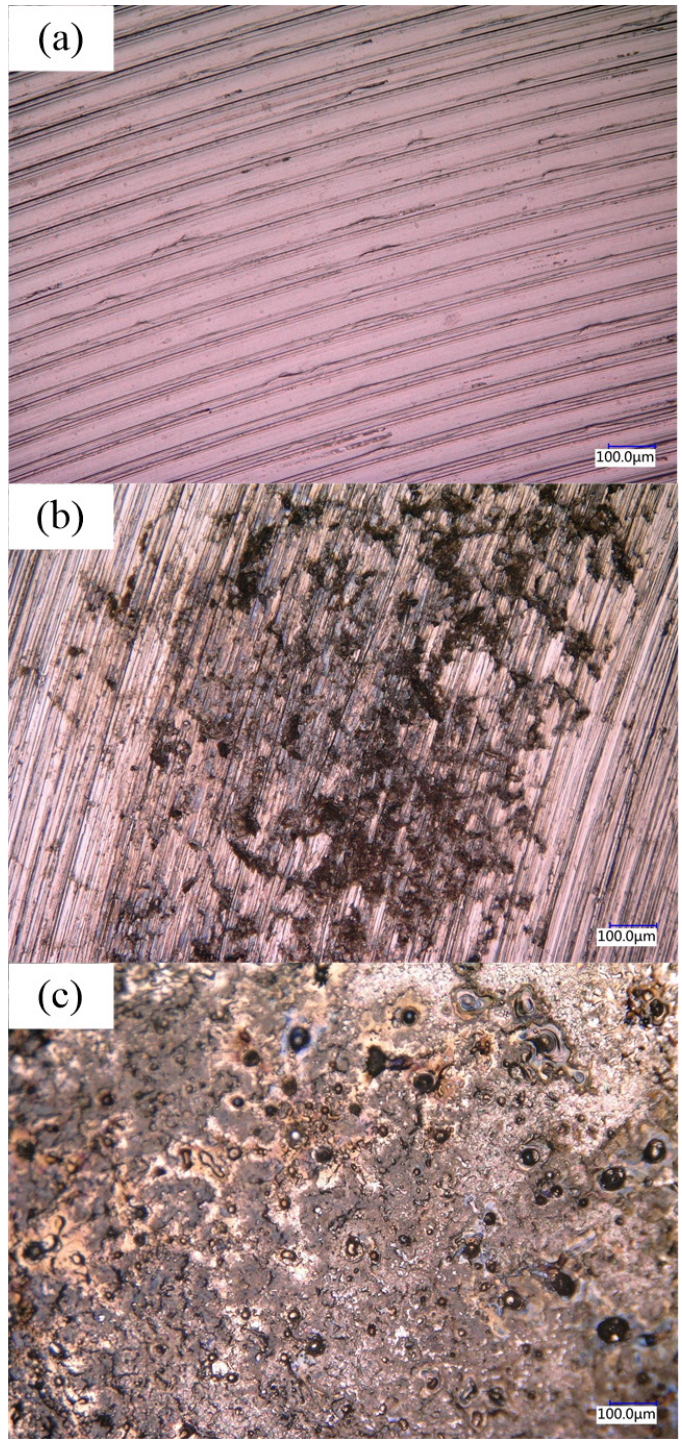

Fig. 15. Micromorphology of trigger electrode after different tested shots. (a) initial state. (b) after 1,000 shots. (c) after 100,000 shots.

The time-delay jitter degradation due to the electrode profile change after 70,000 shots can be explained by the comparation of the micromorphology of the electrode after different number of pulse shots, as shown in Fig. 15. After 1000-shot tests, the surface of the electrode became rough and a large number of tip protrusions appeared (Fig. 15 (b)), which promoted the generation of initial electrons and facilitated the breakdown of the switch, resulting in much lower time delay jitter. This explanation can be supported by another article [38,39]. However, with the increase of shots, the protrusions on the surface of the electrode are relatively uniform and smooth (Fig. 15 (c)). As mentioned above when the number of shots exceeds 70,000 , the time-delay jitter of the switch gradually increases. This shows that from that time onwards, the electrode surface erosion gradually becomes uniform and smooth, and the tip protrusions are 
reduced. Experimental results show that moderate erosion of the electrodes is beneficial in reducing the time-delay jitter of the switch, and excessive erosion increases the time-delay jitter.

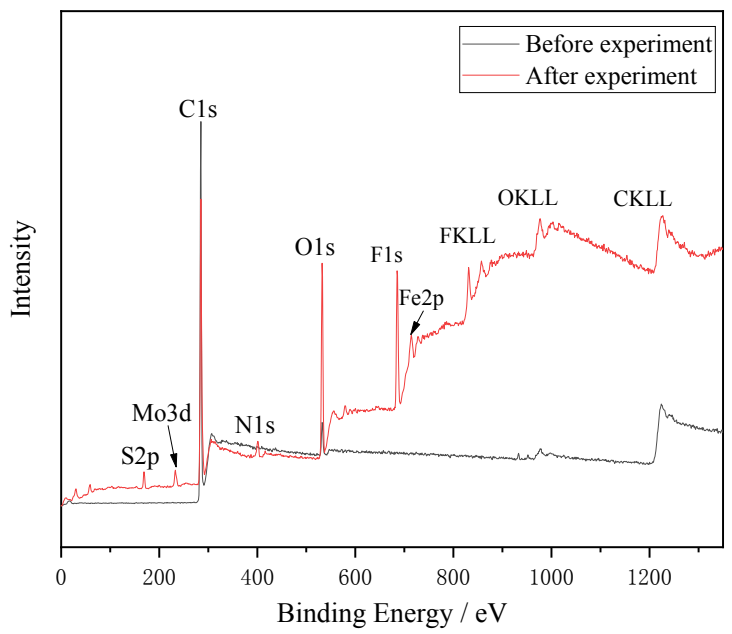

Fig. 16. The full XPS spectrum of the white powder present on the plate insulator surface after 100,000 shots compared with the initial spectrum obtained from the pure PMMA before the tests.

The chemical composition of the white powder present on the surface of the plate insulator was analyzed using X-ray photoelectron spectroscopy (XPS), with the main results presented in Fig. 16. When the surface of the insulator is analyzed after a large number of high-frequency operations, peaks of elements such as $\mathrm{S}, \mathrm{N}$ and $\mathrm{F}$ are clearly present. Further analysis indicates that gases, such as $\mathrm{CO}_{2}, \mathrm{CO}, \mathrm{CF}_{4}$ and $\mathrm{H}_{2} \mathrm{~S}$ were also generated during the discharges. An unexpected, but very important finding is the presence of metallic elements such as Fe and Mo on the surface of the insulator, which may cause its insulation performance to quickly deteriorate.

The presence of fluorides, sulfides and metal particles inside the switch cavity after a certain number of shots can also affect the performance, causing the time jitter to gradually decrease.

\section{CONCLUSIONS}

A compact, repetitive, high-voltage, high-current and low-jitter field-distortion spark gap switch was designed, manufactured and its performances studied in great detail. The size and weight of the unit are $150 \mathrm{~mm} \times 42 \mathrm{~mm}$ and $1.5 \mathrm{~kg}$, respectively.

Simulation results show the switch operates with a field enhancement factor of 1.74. The switch requires a large number of shots for an initial 'forming'. After forming and when filled with $\mathrm{SF}_{6}$ at an absolute pressure of $0.4 \mathrm{MPa}$, the switch static self-breakdown voltage can reach a value in excess of $110 \mathrm{kV}$. The switch has also a wide voltage operating range of $31 \%$ to $90 \%(42 \%$ to $85 \%)$ of the pulsed self-breakdown voltage at a gas pressure of $0.1 \mathrm{MPa}(0.4 \mathrm{MPa})$. When the ratio of operating voltage to self-breakdown voltage is greater than 0.7 , the switch time jitter is less than $3 \mathrm{~ns}$. When the switch operates at a repetition rate of no more than $50 \mathrm{~Hz}$, the time jitter is very stable and varies between $2.0 \mathrm{~ns}$ and $4.0 \mathrm{~ns}$. The experimental results show that if the switch is operated continuously at a current of about $8.5 \mathrm{kA}$ for 100,000 shots, its characteristics remain perfectly stable.

Future plans include increasing the operation life of the switch by using a better erosion-resistant electrode material such as a copper-tungsten alloy.

\section{REFERENCES}

[1] M. Buttram, "Some future directions for repetitive pulsed power," IEEE Trans. Plasma Sci., vol. 30, no. 1, pp. 262-266, Feb. 2002

[2] S. D. Korovin, V. V. Rostov, S. D. Polevin, I. V. Pegel, E. Schamiloglu, M. I. Fuks and R. J. Barker, "Pulsed power-driven high- power microwave sources," Proceedings of the IEEE, vol. 92, no. 7, pp.1082-1095, July 2004

[3] A. A. Kim, B. M. Kovalchuk, V. A. Kokshenev, A. V. Shishlov, N. A. Ratakhin, V. I. Oreshkin, V. V. Rostov, V. I. Koshelev and V. F. Losev, "Review of high-power pulsed systems at Institute of High Current Electronics," Matter and Radiation at Extremes, vol. 1, pp. 201-206, 2016.

[4] G. J. J. Winands, Z. Liu, A. J. M. Pemen, E. J. M. Van Heesch and K. Yan, "Long lifetime, triggered, spark-gap switch for repetitive pulsed power application,” Rev. Sci. Instrum., vol. 76, 085107, 2005.

[5] B. M. Kovalchuk, A. V. Kharlov, E. V. Kumpyak and N. V. Tsoy, "Twoelectrode gas switch with electrodynamical acceleration," Rev. Sci. Instrum., vol. 86, 123504, 2015.

[6] C. Li, L. Lee, B. Chao, Y. L. Liu, B. Yu, Y. F. Ge, L. J. Xie and F. C. Lin, "Study of characteristics and performance optimization of a three-electrode spark gas," IEEE Trans. Dielectr. Electr. Insul., vol. 20, no. 4, pp.1032-1039, Aug. 2013.

[7] Z. Liu, K. Yan, G. J. J. Winands, A. J. M. Pemen, E. J. M. Van Heesch and D. B. Pawelek, "Multiple-gap spark gap switch," Rev. Sci. Instrum., vol. 77, 073501, 2006.

[8] X. B. Cheng, J. L. Liu, B. L. Qian, Z. Chen and J. H. Feng, "Research of a high-current repetitive triggered spark-gap switch and its application," IEEE Trans. Plasma Sci., vol. 38, no. 3, pp.516-522, Mar. 2010.

[9] Yu. B. Smakovskii, Yu. A. Satov, S. V. Khomenko, A. V. Charushin, I. V. Ermilov and V. L. Laptev, "A triggered gas-filled metal-ceramic spark gap with a high current rise rate and stable operation," Instruments and Experimental Techniques, vol. 46, no. 1, pp.45-47, 2003.

[10] K. Yan, E. J. M. van Heesch, S. A. Nair and A. J. M. Pemen, "A triggered spark-gap switch for high-repetition rate high-voltage pulse generation," Journal of Electrostatics, vol. 57, pp.29-33, 2003.

[11] J. M. Lehr, M. D. Abdalla, F. R. Gruner, B. C. Cockreham, M. C. Skipper, S. M. Ahern and W. D. Prather, "Development of a hermetically sealed, high-energy trigatron switch for high repetition rate applications," IEEE Trans. Plasma Sci., vol. 28, no. 5, pp.1469-1475, Oct. 2000.

[12] Y. J. Chen, J. J. Mankowski, J. C. Dickens, J. Walter and M. Kristiansen, "Low-jitter triggered spark gap with high-pressure gas mixtures," IEEE Trans. Plasma Sci., vol. 36, no. 5, pp.2546-2553, Oct. 2008.

[13] H. Watson, "Long-life high-repetition-rate triggered spark gap," IEEE Trans. Plasma Sci., vol. PS-8, no. 3, pp.154-159, Sep. 1980.

[14] High precision spark gap switches, Available: https://www.13t.com.

[15] Specifications for SG-series spark-gap switches, Available: https://www.reb3.com.

[16] A. Kim, S. Frolov, V. Alexeenko, V. Sinebryukhov, M. Mazarakis and F. Bayol, "Prefire probability of the switch type fast LTD," IEEE Pulsed Power Conf., 2009, pp. 565-570.

[17] J. R. Woodworth, J. A. Alexander, W. A. Stygar, L. F. Bennett, H. D. Anderson, M. J. Harden, J. R. Blickem, F. R. Gruner and R. White, "Low inductance switching studies for Linear Transformer Drivers," in IEEE Pulsed Power Conf., Washington, DC, USA, Jun. 2009, pp. 250-254.

[18] J. J. Leckbee, G. E. Pena, M. L. Kiefer, J. A. Alexander, B. S. Stoltzfus, J. L. Brown, H. Wigelsworth, F. E. White and B. Bui, "Comparison of trigger requirements for gas switched for Linear Transformer Drivers," in IEEE Int. Power Modulator and High Voltage Conf., 2014, pp. 93-96.

[19] X. D. Liu, F. J. Sun, T. X. Liang, X. F. Jiang, Q. G. Zhang and A. C. Qiu, "Experimental study on multigap multichannel gas spark closing switch for LTD," IEEE Trans. Plasma Sci., vol. 37, no. 7, pp. 1318-1323, July 2009.

[20] J. B. Jiang, J. Liu, M. H. Liu and M. B. He, "Design and construction of a $\pm 100 \mathrm{kV}$ gas switch for Linear Transformer Drivers," IEEE Trans. Dielectr. Electr. Insul., vol. 22, no. 2, pp. 1292-1297, Apr. 2015.

[21] J. A. Gaudet, R. J. Barker, C. J. Buchenauer, C. Christodoulou, J. Dickens, M. A. Gundersen, R. P. Joshi, H. G. Krompholz, J. F. Kolb, A. Kuthi, M. Laroussi, A. Neuber, W. Nunnally, E. Schamiloglu, K. H. Schoenbach, J. S. Tyo and R. J. Vidmar, "Research issues in developing compact pulsed 
power for high peak power applications on mobile platforms," Proceedings of the IEEE, vol. 92, no. 7, pp. 1144-1165, July 2004.

[22] A. A. Neuber, Y. J. Chen, J. C. Dickens and M. Kristiansen, "A compact, repetitive, $500 \mathrm{kV}, 500 \mathrm{~J}$, Marx generator," IEEE Pulsed Power Conf., 2005, pp. 1203-1206.

[23] A. Sharma, S. kumar, S. Mitra, V. Sharma, A. Patel, A. Roy, T. Menon, K.V. Nagesh and D. P. Chakravarthy, "Development and characterization of repetitive 1-kJ Marx-generator-driven reflex triode system for highpower microwave generation," IEEE Trans. Plasma Sci., vol. 39, no. 5, pp. 1262-1267, May 2011.

[24] F. L. Song, F. Li, B. Z. Zhang, H. T. Gong, Y. Q. Gan and X. Jin, “A compact low jitter high power repetitive long-pulse relativistic electron beam source," Nucl. Instrum. Methods Phys. Res. A, vol. 919, pp. 56-63, 2019.

[25] H. Tang and V. Scuka, "The breakdown mechanism of mid-plane triggered spark gap trigatron," IEEE Trans. Dielectr. Electr. Insul., vol. 3, no. 6, pp. 843-848, Dec. 1996.

[26] A. Saxena, S. Tewari, N. M. Singh, A. Sharma and K. C. Mittal, "Simulation of breakdown mechanism in high power spark gaps filled with nitrogen gas at atmospheric pressure," Radiation Effects \& Defects in Solids, vol. 170, no. 2, pp. 111-120, 2015

[27] S. H. Nam, H. Rahaman, H. Heo and S. S. Park, "Empirical analysis of high pressure $\mathrm{SF}_{6}$ gas breakdown strength in a spark gap switch," IEEE Trans. Dielectr. Electr. Insul., vol. 16, no. 4, pp. 1106 -1110, Aug. 2009.

[28] S. J. MacGregor, S. M. Turnbull, F. A. Tuema and O. Farish, "Factors affecting and methods of Improving the pulse repetition frequency of pulsed-charged and DC-charged high-pressure gas switched," IEEE Trans. Plasma Sci., vol. 25, no. 2, pp. 110-117, Apr. 1997.

[29] S. L. Moran and L. F. Rinehart, "Voltage recovery time of small spark gaps," IEEE Trans. Plasma Sci., vol. PS-10, no. 4, pp. 277-280, Dec. 1982.

[30] Y. Yin, J. L. Liu, H. H. Zhong and J. H. Feng, "Experimental study of the recovery times of spark gap switch with different gases," Plasma Sci. Technol., vol. 10, no. 3, pp. 379-382, Jun. 2008.

[31] J. W. Wu, R. Y. Han, W. D. Ding, H. B. Zhou, Y. F. Liu, Q. J. Liu, Y. Jing and A. C. Qiu, "Electrode erosion characteristics of repetitive longlife gas spark switch under airtight conditions," IEEE Trans. Plasma Sci., vol. 43, no. 10, pp. 3425-3433, Oct. 2015.

[32] Y. Liu, Z. Y. Li, Q. P. Luo, Y. B. Han, Q. Zhang and F. C. Lin, "Comparison and evaluation of electrode erosion under high-pulsed current discharges in air and water mediums," IEEE Trans. Plasma Sci., vol. 44, no. 7, pp. 1169-1177, July 2016.

[33] J. M. Koutsoubis and S. J. MacGregor, "Electrode erosion and lifetime performance of a high repetition rate, triggered, corona-stabilized switch in air," J. Phys. D: Appl. Phys., vol. 33, pp. 1093-1103, 2000.

[34] X. A. Li, X. D. Liu, X. Q. Gou, F. H. Zeng and Q. G. Zhang, "Degradation of performance due to electrode erosion in field distortion gas switch in long-term repetitive operation," IEEE Trans. Plasma Sci., vol. 42, no. 10, pp. 3064-3069, Oct. 2014.

[35] V. Nemchinsky, "Cathode erosion in a high-pressure high-current arc: calculations for tungsten cathode in a free-burning argon arc," J. Phys. D: Appl. Phys., vol. 45, 135201, 2012.

[36] A. L. Donaldson, M. Kristiansen, A. Watson, K. Zinsmeyer and E. Kristiansen, "Electrode erosion in high-current, high-energy transient arcs," IEEE Trans. Magnetics, vol. Mag-22, no. 6, pp. 1441-1447, Nov. 1986.

[37] J. M. Koutsoubis, S. J. MacGregor and S. M. Turnbull, "Triggered switch performance in $\mathrm{SF}_{6}$, air, and an $\mathrm{SF}_{6}$ /air mixture," IEEE Trans. Plasma Sci., vol. 27, no. 1, pp. 272-281, Feb. 1999.

[38] J. Y. Geng, J. H. Yang, X. B. Cheng, X. Yang and R. Chen, "The development of high-voltage repetitive low-jitter corona stabilized triggered switch,” Rev. Sci. Instrum., vol. 89, 044705, 2018.

[39] A. Pedersen, "The effect of surface roughness on breakdown in $\mathrm{SF}_{6}$," IEEE Trans. Power Apparatus and systems, vol. PAS-94, no. 5, pp. 17491754, September/October 1975.

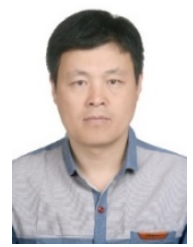

Falun Song was born in Shandong province, China, in 1977. $\mathrm{He}$ received the B.S. degree from Liaocheng Normal University, Liaocheng, China, in 2000 and the Ph.D. degree from University of Science and Technology of China, Anhui, China, in 2005. He is currently an Associate Professor with the Institute of Applied Electronics, China Academy of Engineering Physics, where he works on pulsed power and plasma immersion ion implantation technology.

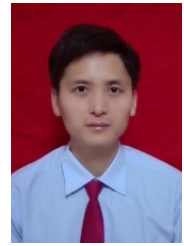

Fei Li was born in Shanxi province, China in 1987. He received the B.S. degree in 2009 and the Ph.D. degree in 2014 from Lanzhou University, Lanzhou, China. His research interests are pulsed power technology and material surface treatment technology.

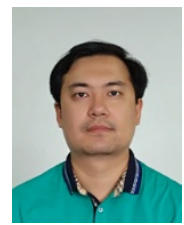

Beizhen Zhang was born in Heilongjiang province, China, in 1982. He received his B.S. degree from Jilin University, Jilin, China, in 2005. His research interests are pulsed power technology and mechanical structural design.

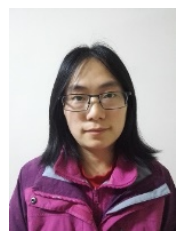

Chunxia Li was born in Sichuan province, China, in 1983. She graduated in 2005 from Tsinghua University, Beijing, China. Her research interests are pulsed power technology and electron beam diagnostic technology.

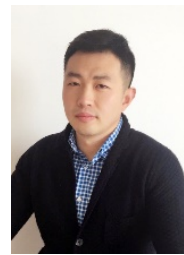

Ganping Wang was born in Shandong province, China, in 1983. He received the B.S. degree from the University of Science and Technology of Xi' an, Xi' an, China, in 2005 and the M.S. degree in physics of Wireless Electronics from the Graduate School of China Academy of Engineering Physics (CAEP), Beijing, China, in 2009. He is currently working toward the Ph.D. degree in physics of Wireless Electronics from Graduate school of CAEP. His research interests are high-power pulsed power technology.

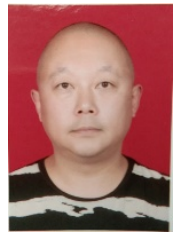

Haitao Gong was born in Sichuan province, China, in 1973. $\mathrm{He}$ is a skilled worker who has been engaged in research on the pulsed power sources for more than twenty-three years.

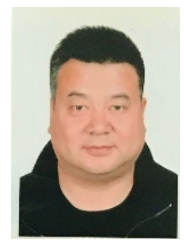

Yanqing Gan was born in Gansu province, China, in 1970. He has been engaged in research on pulsed power sources for more than twenty-seven years. He is presently working on pulsed power measurement technology and plasma diagnostic technology.

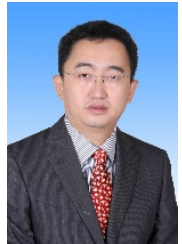

Xiao Jin was born in Zhejiang province, China, in 1969. He received the B.S. degree from Tsinghua University, Beijing, China, in 1992 and the Ph.D. degree from the Graduate School of China Academy of Engineering Physics, Beijing, in 2002, and became a professor in 2006. His research interests are the pulsed power technology, high power microwave technology, and free-electron laser technology.

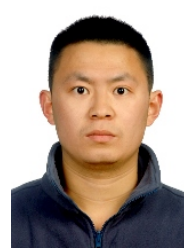

Mingdong Zhu was born in Sichuan province, China, in 1989. He received the B. S. and M. S. degrees from Harbin Institute of Technology (HIT), Harbin, China, in 2011 and 2014, respectively. He is currently working toward the $\mathrm{Ph}$. D. degree in China State Key Laboratory of Advanced Welding and Joining at HIT. He has been working on plasma immersion ion implantation technology. 


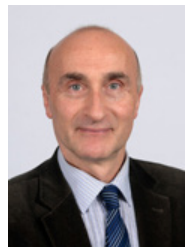

Bucur M. Novac (M'06 - SM'08) received the M.Sc. and Ph.D. degrees in 1977 and 1989, respectively, both from the University of Bucharest. He joined Loughborough University, UK in 1998 and is currently Professor of Pulsed Power. His research interests include compact and repetitive highpower systems, explosively and electromagnetically driven magnetic flux compression generators and their applications, electromagnetic launchers, ultrafast magneto and electro-optic sensors and 2-D modeling of pulsed-power systems. He has co-authored two books on explosive pulsed power and has published more than 200 refereed papers and conference contributions.

Prof. Novac is a voting member of the Pulsed Power Science \& Technology Committee in the IEEE Nuclear and Plasma Science Society. He is also a member of the International Steering Committees for both the MEGAGAUSS Conferences and for the Euro-Asian Pulsed Power Conferences. He is also member of the organizing committee for the IEEE International Power Modulator and High Voltage Conference and co-chairman of the UK Pulsed Power Symposium. Prof. Novac is a Chartered Engineer a Fellow of the Royal Academy of Engineering and of the Institution of Engineering and Technology (IET), UK.

Ivor R. Smith received B.Sc. and Ph.D. degrees from the University of Bristol, Bristol, U.K., after completing a student apprenticeship at the Witton Works of the General Electric Company, and the D.Sc. degree from the University of Birmingham, Birmingham, U.K., for his continued research contribution. He was a Lecturer in electrical engineering with the University of Birmingham and was subsequently promoted to Lecturer and Reader. He then moved to Loughborough University, Leicestershire, U.K., as a Professor of electrical power engineering and served as the Head of Department, Dean of Engineering, and ProVice Chancellor. He has been active in research in many aspects of the production, conditioning, and utilization of large pulses of electrical energy, and his work has brought in very substantial funding from a wide range of sponsors. Dr. Smith is a Chartered Engineer, a Fellow of the Institution of Engineering and Technology and the Royal Academy of Engineering. 